8

\title{
Role of Annona muricata (L.) in Oxidative Stress and Metabolic Variations in Diabetic and Gamma-irradiated Rats
}

\author{
E.T. ${ }^{1}$ Mohamed, M. E. El-Sayed Mahdy ${ }^{2}$, G.A.M.Singer ${ }^{2}$, Shereen M. ElKiki $^{1}$ \\ and M. S.Elias ${ }^{1^{*}}$ \\ Health Research Department, National Center for Radiation Research and \\ Technology, Atomic Energy Authority and ${ }^{2}$ Biochemistry Department, Faculty of \\ Science,Helwan University, Cairo, Egypt
}

\begin{abstract}
A . MURICATA L., commonly known as graviola, is a plant widely distributed throughout

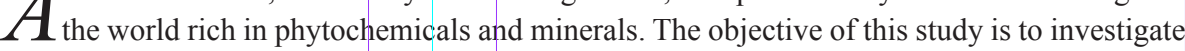
the influence of A.muricata on oxidative stress in the liver, kidney and pancreas along with variations in glucose, insulin, lipid profile, liver and kidney functions in diabetic (DM) and $\gamma$-irradiated rats (IRR).Diabetes was induced by a single intraperitoneal injection of streptozotocin $(65 \mathrm{mg} / \mathrm{Kg}$ body weight). Irradiation was performed as a whole body $\gamma$-irradiation ( $5 \mathrm{~Gy}$ ) administered in a single acute dose. A.muricata leaves aqueous extract $(100 \mathrm{mg} / \mathrm{Kg}$ body weight) was administered via gavages during 2 weeks to diabetic rats or during 2 weeks before $\gamma$-irradiation. Diabetic and irradiated rats received A.muricata during 2 weeks before irradiation. Animals were sacrificed 24 hours post irradiation and/or A.muricata trearment. A.muricata treatment has significantly attenuated hyperglycemia, hypoinsulinemia and dyslipidemia, and reduced the increase of serum alanine and aspartate amino transferase activities and serum urea and creatinine levels. The amelioration in metabolic variations was associated to significant improvement of oxidative stress in tissues notified by a higher superoxide dismutase (SOD) activity and glutathione (GSH) content and a lower malondialdehyde (MDA) content.It could be suggested that the synergistic relationship between the different elements found in the leaf of A.muricata could be beneficial in ameliorating liver and kidney functions and correcting metabolic variations associated with oxidative stress in the liver, kidney and pancreas.
\end{abstract}

Keywords: Diabetes, streptozotocin, $\gamma$-irradiation, A.muricata, oxidative stress, metabolic variations.

\section{Introduction}

In recent years, an interest in the phytochemistry of plant has been sparked. The genus Annona belonging to the Custard Apple family, Annonaceae, is widespread in the tropical regions of the world and includes the species Annona muricata (Linn). The phytochemical analysis conducted on A.muricata dried and powdered leaves revealed the presence of flavonoids, alkaloids, tannins, saponins and reducing sugars. Mineral analysis showed the presence of potassium (363.05 mg/kg), calcium $(11,183.50 \mathrm{mg} / \mathrm{kg})$, sodium (694.86 mg/kg), magnesium $(9,619 \mathrm{mg} / \mathrm{kg})$, iron (139.50 mg/kg), zinc (8.34 mg/kg), manganese (8.25 $\mathrm{mg} / \mathrm{kg})$, chromium $(3.75 \mathrm{mg} / \mathrm{kg})$, copper $(14.25$ $\mathrm{mg} / \mathrm{kg}$ ) and cadmium $(5.49 \mathrm{mg} / \mathrm{kg}$ ) (Usunomena and Paulinus, 2015).

Attention to the role of $A$. muricata in human health has increased following the discovery that it possesses anticancer (Asare et al.,2015;
Coria-Tellez et al., 2016), antidiabetic(Ahalya et al., 2014), anti-inflammatory (Isholaet al., 2014), hepatoprotective(Art hur et al., 2012), antioxidantandfree radical scavenging activities (George et al., 2015; Coria-Tellez et al., 2016). The plant A. muricata was even described as a "Miracle Fruit" (Patel and Patel, 2016).

Diabetes mellitus (DM) is a chronic disease characterized by hyperglycemia due to defects in insulin production and/or action (American Diabetes Association, 2009). Along with hyperglycemia,diabetes is associated with degenerative complications in many organs including the pancreas, liver, kidney, heart, muscles, and eyes.Oxidative stress was reported to have a central role in the onset of DM and its complications (Wang et al., 2015).

On the other hand, exposure to ionizing radiation has become inevitable due to the increase in the development of nuclear 
technology.Experimental studies demonstrated that receiving an acute dose of ionizing radiation induces oxidative stress associated with metabolic alterations (Saada et al., 2016).Efficient defense and repair mechanisms exist in living cells to protect against oxidant species. Superoxide dismutase (SOD) catalyzes the reduction of superoxide anion to hydrogen peroxide $\left(\mathrm{H}_{2} \mathrm{O}_{2}\right)$, which is broken down by catalase and glutathione peroxidase (GSH-Px) (Sun et al., 1998). However under abnormal conditions, the antioxidant system may not be adequate to protect from oxidative stress and metabolic alterations.

The objective of this study is to investigate if A. Muricata leaves aqueous extract would alleviate oxidative stress associated with some metabolic alteration in diabetic, $\gamma$-irradiated and diabetic- $\gamma$-irradiated rats.

\section{Materials and Methods}

Animals

Healthy male adult albino rats SpragueDawley $(10 \pm 2$ weeks old; $120 \pm 20 \mathrm{~g})$ were obtained from the Nile Company for Pharmaceuticals and Chemical Industries, Cairo, Egypt.Animals were housed under standard laboratory conditions of ventilation, temperature and humidity in specially-designed plastic cages along the experiment period. The rats were fed with commercially available rat pelleted diet, containing all the required nutritive elements. Water and food were provided ad libitum throughout the study.Experimental analyses were performed in the morning at 11:00 \pm 1.00 hour.All animal procedures were approved by the Ethics Committee of the National Research Center conformed to the "Guide for the care and use of Laboratory Animals" published by the National Institutes of Health (NIH publication No. 85-23, revised 1996).

\section{Induction of Diabetes}

Streptozotocin (STZ), purchased from Sigma Chemical Company, St. Louis Missouri, USA, in the form of $1 \mathrm{~g}$ vial was administered to rats in a single intraperitoneal (i.p.) injection at a dose of 65 $\mathrm{mg} / \mathrm{Kg}$ body weight, dissolved in freshly prepared $0.1 \mathrm{M}$ cold sodium citrate buffer ( $\mathrm{pH} 4.5)$ (Erejuwa et al., 2011). Owing to the high destructive power of STZ on pancreatic $\beta$-cells and massive release of insulin, $10 \%$ sucrose solution was allowed to rats for the next 24hours to avoid hypoglycemic shock (Gandhi and Sasikumar, 2012). Monitoring of blood glucose levels was performed 72 hours Egypt. J. Rad. Sci. Applic., Vol. 30, No.1(2017) after STZ administration, using an Accu-check blood glucose meter (Roche Diagnostics, Basel, Switzerland) in tail vein blood. Rats with blood glucose levels $\geq 250 \mathrm{mg} / \mathrm{dL}$ were considered diabetics and selected for this study.

\section{Radiation Treatment}

A whole-body $\gamma$-irradiation of rats with $5 \mathrm{~Gy}$, applied as a single acute dose, ata dose rate of 0.5 Gy/minute was carried out at the National Center for Radiation Research and Technology (NCRRT), Nasr City, Cairo, Egypt. The source of radiation was a Canadian Gamma cell-40 (Cesium-137), which ensured a homogeneous dose distribution all over the irradiation tray.

\section{Annona muricataTreatment}

Annona muricata leaf aqueous extract was purchased from USA, under the trade name of Graviola 750 in the form of capsules. The content was dissolved in saline and administered to rats daily via gavages at doses of $100 \mathrm{mg} / \mathrm{Kg}$ body weight/day (in $1 \mathrm{~m} \mathrm{~L}$ saline) during 14 consecutive days according to Florence et al. (2014).

\section{Experimental Design}

A total of 80 rats were divided into 8 groups (10 rats per group):1- Control group: Rats given $1 \mathrm{~mL}$ saline during 14 days via gavages. 2- A. muricata group: Rats daily supplemented with Annona muricata (100 mg/Kg body weight/day) during 14 days via gavages.3- DM group: Diabetic rats given $1 \mathrm{~mL}$ saline daily during 14 days via gavages.4$\mathrm{DM}+A$. muricata group: Diabetic rats given $A$. muricata $(100 \mathrm{mg} / \mathrm{Kg}$ body weight/day) daily during14 days via gavages.5- IRR group: Rats given $1 \mathrm{~mL}$ saline during 14 days via gavages then exposed to a whole body $\gamma$-irradiationat a dose rateof 5Gy.6- $A$. muricata + IRR group: Rats given A. muricata (100 mg/Kg body weight/day) daily during 14 days via gavages before $\gamma$-irradiation (a whole body exposure at 5 Gy).7- DM + IRR group: Diabetic rats given saline via gavages during 14 days then whole body $\gamma$-irradiated with 5 Gy.8- DM + A. muricata+ IRR group: Diabetic rats given $A$. muricata via gavages during 14 days then whole body $\gamma$-irradiated with 5 Gy.

\section{Collection and Processing of Blood and Tissue} Samples:

The animals were sacrificed $24 \mathrm{hr}$. postirradiation or $A$. muricata treatment after a fasting period of $12 \mathrm{hr}$. Blood samples were obtained via heart puncture by sterilized syringe and the serum obtained after centrifugation at $3000 \mathrm{rpm}$ for $15 \mathrm{~min}$ 
(Cent rifuge, PLC-036, Taiwan). The liver, kidney and pancreas were quickly excised washed in ice-cold saline. A homogenate was prepared in $0.9 \%$ saline $(20 \% \mathrm{~W} / \mathrm{V})$ using digital homogenizer (WiseTis HG-15D, Germany) and the homogenates were stored at $-20^{\circ} \mathrm{C}$ until a further biochemical analysis.

\section{Biochemical Analysis}

Chemicals and reagents were purchased from Sigma-Aldrich, St Louis, MO, USA otherwise mentioned. Measurement of absorbance was performed using a T60 UV/VIS spectrophotometer, PG instruments, London, UK. Tissue homogenates were obtained using digital homogenizer, WiseTis HG-15D, Germany. Centrifugation was carried out using cooling centrifuge, Hettich, MIKRO 22R, Germany.

\section{Estimation of Metabolic Variations}

Glucose was determined using diagnostic kit purchased from Spectrum Egypt according to the method described by Trinder (1969). Insulin was determined using enzyme-linked immunosorbent assay (ELISA) according to Clark and Hales (1994). The variation of serum lipid profile was carried out using Spectrum Egypt diagnostic kit for the estimation of serum triglycerides (TG) (Fossati and Prencipe, 1982), total cholesterol (Richmond,1973) and high-density lipoproteincholesterol (HDL-C) (Friedewald et al.,1972). Low-density lipoprotein-cholesterol (LDL-C) was calculated using the formulaof Friedewald et al. (1972):LDL-C level = Total Cholesterol (TG/5) - HDL-C.The atherogenic index (AI); a logarithmic ratio between the concentrations of TG to HDL-C [Log (TG/HDL-C)] used for the diagnosis and prognosis of cardiovascular disease (CVD) (Dobiasova, 2006)was calculated using the online Calculator of atherogenic risk.

$\mathrm{AI}<0.11$ was considered a low risk for CVD; AI ( 0.11 to 0.21$)$ : was considered a medium risk for CVD; AI >0.21 was considered a high risk for CVD.

Assessment of Liver and Kidney Functions

Liver function was assayed by the measurement of serum alanine aminotransferase (ALT) and aspartate aminotransferase (AST) activities according to Reitman and Frankel (1957). Kidney function was evaluated by measuring serum creatinine and urea levels according to Henry et al. (1974) and Patton and Crouch(1977), respectively.
Assessment of Oxidative Stress

Lipid peroxidation was determined as described by Yoshioka et al. (1979) based on the determination of malondialdehyde (MDA), an end product of lipid peroxidation, which can react with thiobarbituric acid in acidic medium to yield a pink colored trimethine complex which wasmeasured at absorbance of $532 \mathrm{~nm}$.Superoxide dismutase activity (SOD) was determined according to the method of Kakkar et al.(1984). Glutathione (GSH)content was determined according to the method of Beutler et al. (1963).

\section{Statistical Analysis}

All values are represented as Mean \pm Standard deviation. All groups were compared by one-way analysis of variance (ANOVA) and post hoc multiple comparisons were done with LSD test in SPSS/PC software program (version 20.0; SPSS Inc., Chicago, IL, USA) to determine the differences between the studied groups. Differences were considered statistically significant at $\mathrm{p} \leq 0.05$ and highly significant at $\mathrm{P} \leq$ 0.01 .

\section{Results}

Supplementation of rats with the aqueous extract of $A$. muricat aleaves (100 mg/Kg b.wt/ day) daily via gavages during 14 days has not induced significant changes in SOD, GSH and MDA levels in the liver, kidney and pancreas, compared to control(Tables 1\&2). SOD activity and GSH content were lower and MDA level was higher in the liver, kidney and pancreas of diabetic (DM), $\gamma$-irradiated (IRR) and diabetic- $\gamma$ irradiated (DM+IRR) rats compared to contro 1.A. muricata treatment has significantly ameliorated oxidative stress by increasing SOD and GSH and decreasing MDA as recorded in DM+A.muricata, A.muricata $+\mathrm{IRR}$ and $\mathrm{DM}+$ A.muricata $+\mathrm{IRR}$ groups (Tables 1\&2).

The supplementation of rats with the aqueous extract of $A$. muricata leaves $(100 \mathrm{mg} / \mathrm{Kg}$ b.wt/ day) daily via gavages during 14 days had no significant effect on glucose, insulin and lipid profile (Table 3).The level of glucose was significantly higher and insulin was significantly lower in the diabetic (DM), $\gamma$-irradiated (IRR) and diabetic- $\gamma$-irradiated (DM+ IRR) groups, compared to control. A. muricata treatment restored glucose and insulin to the normal level (Table 3).The levels of triglycerides, cholesterol and LDL-C were significantly higher and HDL-C 
was significantly lower in the diabetic (DM), $\gamma$-irradiated (IRR) and diabetic- $\gamma$-irradiated (DM+ IRR) groups, compared to control (Table 3).A. muricata treatment has significantly ameliorated the variations in lipid profile by reducing triglycerides, cholesterol and LDL-C and increasing HDL-C (Table 2). The atherogenic index (AI) in diabetic rats was 0.113 indicating a low risk of CVD while in diabetic irradiated rats AI was 0.229 indicatinga high risk. A. muricata treatment has significantly improved the AI in diabetic and diabetic irradiated rats (Table 3 ).
The supplementation of rats with the aqueous extract of $A$. muricata leaves $(100 \mathrm{mg}$ / $\mathrm{Kg}$ b.wt/day) daily via gavages during 14 days had no effect on AST and ALT activities, urea and creatinine levels, compared to control (Table 4). AST and ALT activities, urea and creatinine levels were significantly elevated in the diabetic (DM), $\gamma$-irradiated (IRR) and diabetic- $\gamma$-irradiated (DM+IRR) rats, compared to control. A. muricata treatment has significantly lowered ALT and AST activities as well as urea and creatinine levels (Table 4).

TABLE 1. Influence of Annona muricata (AM) on antioxidants in different tissues of diabetic (DM), $\gamma$-irradiated (IRR) and diabetic- $\gamma$-irradiated (DM+IRR) rats.

\begin{tabular}{|c|c|c|c|c|c|c|c|c|}
\hline Rat Groups & Control & $\mathbf{A M}$ & DM & $\begin{array}{c}\text { DM } \\
+\mathbf{A M}\end{array}$ & IRR & $\begin{array}{c}\text { AM } \\
+ \text { IRR }\end{array}$ & $\begin{array}{c}\text { DM } \\
+ \text { IRR }\end{array}$ & $\begin{array}{c}\text { DM } \\
+ \text { AM } \\
+ \text { IRR }\end{array}$ \\
\hline \multicolumn{9}{|c|}{ Superoxide dismutase (U/g tissue) } \\
\hline Liver & $\begin{array}{l}36.33 \\
\pm 0.52\end{array}$ & $\begin{array}{l}36.58 \\
\pm 0.49\end{array}$ & $\begin{array}{c}20.26 \\
\pm 2.76 \\
(-44 \%) \\
a^{* *}\end{array}$ & $\begin{array}{c}30.08 \\
\pm 0.92 \\
(-17 \%) \\
b^{* *}\end{array}$ & $\begin{array}{c}28.17 \\
\pm 1.17 \\
(-23 \%) \\
a^{* *}\end{array}$ & $\begin{array}{c}30.60 \\
\pm 4.73 \\
(-16 \%) \\
b^{* *}\end{array}$ & $\begin{array}{c}28.47 \\
\pm 0.93 \\
(-22 \%) \\
a^{* *}\end{array}$ & $\begin{array}{c}31.79 \\
\pm 0.34 \\
(-13 \%) \\
b^{* *}\end{array}$ \\
\hline Kidney & $\begin{array}{l}32.00 \\
\pm 3.43\end{array}$ & $\begin{array}{l}30.92 \\
\pm 0.49\end{array}$ & $\begin{array}{c}23.50 \\
\pm 1.38 \\
(-27 \%) \\
a^{* *}\end{array}$ & $\begin{array}{c}30.08 \\
\pm 1.11 \\
(-6 \%) \\
b^{* *}\end{array}$ & $\begin{array}{c}29.00 \\
\pm 0.89 \\
(-9 \%) \\
a^{*}\end{array}$ & $\begin{array}{c}31.58 \\
\pm 1.36 \\
(-1 \%) \\
b^{*}\end{array}$ & $\begin{array}{c}25.83 \\
\pm 0.41 \\
(-19 \%) \\
a^{* *}\end{array}$ & $\begin{array}{c}29.10 \\
\pm 0.86 \\
(-9 \%) \\
b^{*}\end{array}$ \\
\hline Pancreas & $\begin{array}{c}7.35 \\
\pm 0.07\end{array}$ & $\begin{array}{c}7.14 \\
\pm 0.21\end{array}$ & $\begin{array}{c}6.13 \\
\pm 0.68 \\
(-17 \%) \\
a^{* *}\end{array}$ & $\begin{array}{c}6.96 \\
\pm 0.28 \\
(-5 \%) \\
b^{*}\end{array}$ & $\begin{array}{c}5.87 \\
\pm 0.80 \\
(-20 \%) \\
a^{* *}\end{array}$ & $\begin{array}{c}7.1 \\
\pm 0.26 \\
(-3 \%) \\
b^{* *}\end{array}$ & $\begin{array}{c}6.16 \\
\pm 0.96 \\
(-16 \%) \\
a^{* *}\end{array}$ & $\begin{array}{c}7.26 \\
\pm 0.12 \\
(-1 \%) \\
b^{*}\end{array}$ \\
\hline \multicolumn{9}{|c|}{ Glutathione ( $\mathrm{mg} / \mathrm{g}$ tissue) } \\
\hline Liver & $\begin{array}{l}20.73 \\
\pm 3.04\end{array}$ & $\begin{array}{l}19.52 \\
\pm 1.05\end{array}$ & $\begin{array}{c}6.46 \\
\pm 0.96 \\
(-69 \%) \\
a^{* *}\end{array}$ & $\begin{array}{c}19.28 \\
\pm 1.07 \\
(-7 \%) \\
b^{* *}\end{array}$ & $\begin{array}{c}7.46 \\
\pm 0.61 \\
(-64 \%) \\
a^{* *}\end{array}$ & $\begin{array}{c}21.35 \\
\pm 0.63 \\
(-3 \%) \\
b^{* *}\end{array}$ & $\begin{array}{c}7.55 \\
\pm 1.13 \\
(-64 \%) \\
a^{* *}\end{array}$ & $\begin{array}{c}11.80 \\
\pm 1.41 \\
(-43 \%) \\
\mathrm{b}^{* *}\end{array}$ \\
\hline Kidney & $\begin{array}{l}25.02 \\
\pm 2.26\end{array}$ & $\begin{array}{l}23.67 \\
\pm 4.50\end{array}$ & $\begin{array}{c}11.8 \\
\pm 0.94 \\
(-53 \%) \\
a^{* *}\end{array}$ & $\begin{array}{c}24.04 \\
\pm 2.15 \\
(-4 \%) \\
b^{* *}\end{array}$ & $\begin{array}{c}10.71 \\
\pm 0.95 \\
(-57 \%) \\
a^{* *}\end{array}$ & $\begin{array}{c}24.0 \\
\pm 2.94 \\
(-4 \%) \\
b^{* *}\end{array}$ & $\begin{array}{c}9.86 \\
\pm 0.80 \\
(-61 \%) \\
a^{* *}\end{array}$ & $\begin{array}{c}21.35 \\
\pm 1.70 \\
(-15 \%) \\
b^{* *}\end{array}$ \\
\hline Pancreas & $\begin{array}{c}7.48 \\
\pm 0.68\end{array}$ & $\begin{array}{c}7.72 \\
\pm 1.48\end{array}$ & $\begin{array}{c}5.59 \\
\pm 0.22 \\
(-25 \%) \\
a^{* *}\end{array}$ & $\begin{array}{c}7.60 \\
\pm 0.70 \\
(+2 \%) \\
\mathrm{b}^{* *}\end{array}$ & $\begin{array}{c}6.56 \\
\pm 0.20 \\
(-12 \%) \\
a^{*}\end{array}$ & $\begin{array}{c}7.58 \\
\pm 0.43 \\
(+1 \%) \\
b^{*}\end{array}$ & $\begin{array}{c}6.08 \\
\pm 0.63 \\
(-19 \%) \\
a^{*}\end{array}$ & $\begin{array}{c}7.70 \\
\pm 0.31 \\
(+3 \%) \\
\mathrm{b}^{* *}\end{array}$ \\
\hline
\end{tabular}

Data are expressed as Mean \pm Standard Deviation ( $\mathrm{n}=10$ ). Numbers between brackets show the percentage of change from the respective control value. a: significance vs control. b: significance vs respective DM, IRR and DM+IRR groups not treated with A muricata. *: significant at $\mathrm{P} \leq 0.05 ; * *$ : highly significant at $\mathrm{P} \leq 0.01$ 
TABLE 2. Influence of Annona muricata (AM) on lipid peroxidation marker Malondialdehyde (nmol/g tissue) in different tissues of diabetic (DM), $\gamma$-irradiated (IRR) and diabetic- $\gamma$-irradiated (DM+IRR) rats.

\begin{tabular}{|c|c|c|c|c|c|c|c|c|}
\hline Rat Groups & Control & $\overline{\mathbf{A M}}$ & DM & $\begin{array}{c}\text { DM } \\
+\mathbf{A M}\end{array}$ & IRR & $\begin{array}{c}\text { AM } \\
+ \text { IRR }\end{array}$ & $\begin{array}{c}\text { DM } \\
+ \text { IRR }\end{array}$ & $\begin{array}{c}\text { DM } \\
+\mathbf{A M} \\
+ \text { IRR }\end{array}$ \\
\hline Liver & $\begin{array}{c}139.0 \\
\pm 01.90\end{array}$ & $\begin{array}{c}134.8 \\
\pm 09.52\end{array}$ & $\begin{array}{c}247.8 \\
\pm 14.33 \\
(78 \%) \\
a^{* *}\end{array}$ & $\begin{array}{c}160.5 \\
\pm 14.40 \\
(16 \%) \\
b * *\end{array}$ & $\begin{array}{c}243.0 \\
\pm 11.54 \\
(75 \%) \\
a^{* *}\end{array}$ & $\begin{array}{c}167.2 \\
\pm 12.40 \\
(20 \%) \\
b^{* *}\end{array}$ & $\begin{array}{c}345.7 \\
\pm 11.06 \\
(149 \%) \\
a^{* *}\end{array}$ & $\begin{array}{c}260.2 \\
\pm 15.29 \\
(87 \%) \\
b^{* *}\end{array}$ \\
\hline Kidney & $\begin{array}{c}170.7 \\
\pm 17.26\end{array}$ & $\begin{array}{c}174.2 \\
\pm 10.76\end{array}$ & $\begin{array}{c}610.2 \\
\pm 18.83 \\
(258 \%) \\
a^{* *}\end{array}$ & $\begin{array}{c}419.3 \\
\pm 21.40 \\
(146 \%) \\
b^{* *}\end{array}$ & $\begin{array}{c}498.5 \\
\pm 10.31 \\
(192 \%) \\
a^{* *}\end{array}$ & $\begin{array}{c}327.2 \\
\pm 11.70 \\
(92 \%) \\
b^{* *}\end{array}$ & $\begin{array}{c}974.2 \\
\pm 37.86 \\
(471 \%) \\
a^{* *}\end{array}$ & $\begin{array}{c}569.3 \\
\pm 17.51 \\
(234 \%) \\
b * *\end{array}$ \\
\hline Pancreas & $\begin{array}{l}187.3 \\
\pm 9.75\end{array}$ & $\begin{array}{l}195.7 \\
\pm 6.92\end{array}$ & $\begin{array}{c}299.5 \\
\pm 13.90 \\
(+60 \%) \\
a^{* *}\end{array}$ & $\begin{array}{c}189.2 \\
\pm 9.17 \\
(+1 \%) \\
b * *\end{array}$ & $\begin{array}{c}231.7 \\
\pm 13.44 \\
(+24 \%) \\
\text { a** }\end{array}$ & $\begin{array}{c}183.7 \\
\pm 11.83 \\
(-2 \%) \\
b * *\end{array}$ & $\begin{array}{c}337.2 \\
\pm 7.52 \\
(+80 \%) \\
a * *\end{array}$ & $\begin{array}{c}257.8 \\
\pm 10.03 \\
(+38 \%) \\
b * *\end{array}$ \\
\hline
\end{tabular}

Data are expressed as Mean \pm Standard Deviation $(n=10)$. Numbers between brackets showthe percentage of change from the respective control value. a:significance vs control. b:significance vs respective DM, IRR and DM + IRR groups not treated with Amuricata. *: significant at $\mathrm{P} \leq 0.05$; **: highly significant at $\mathrm{P} \leq 0.01$

TABLE 3. Influence of Annona muricata(AM) on some metabolic variations in the serum of diabetic (DM), $\gamma$-irradiated (IRR) and diabetic- $\gamma$-irradiated (DM+IRR) rats.

\begin{tabular}{|c|c|c|c|c|c|c|c|c|}
\hline $\begin{array}{c}\text { Rat } \\
\text { Groups }\end{array}$ & $\mathrm{C}$ & $\mathbf{A M}$ & DM & $\begin{array}{c}\text { DM } \\
+\mathbf{A M}\end{array}$ & IRR & $\begin{array}{c}\text { AM } \\
+ \text { IRR }\end{array}$ & $\begin{array}{c}\text { DM } \\
+ \text { IRR }\end{array}$ & $\begin{array}{c}\text { DM } \\
+ \text { AM } \\
+ \text { IRR }\end{array}$ \\
\hline Glucose & 80.29 & 81.33 & 281.4 & 78.13 & 131.10 & 86.43 & 145.17 & 85.33 \\
\hline $\mathrm{mg} / \mathrm{dL}$ & \pm 5.91 & \pm 6.03 & $\begin{array}{l} \pm 26.79 \\
(250 \%)\end{array}$ & $\begin{array}{c} \pm 11.18 \\
(-3 \%)\end{array}$ & $\begin{array}{c} \pm 7.5 \\
(63 \%)\end{array}$ & $\begin{array}{c} \pm 12.78 \\
(7 \%)\end{array}$ & $\begin{array}{l} \pm 21.2 \\
(80 \%)\end{array}$ & $\begin{array}{c} \pm 6.32 \\
(6 \%)\end{array}$ \\
\hline Insulin & 5.72 & 5.88 & $\begin{array}{l}\mathrm{a}^{* *} \\
4.12\end{array}$ & $\begin{array}{l}\mathrm{b} * * \\
5.38\end{array}$ & $\begin{array}{l}\mathrm{a}^{* *} \\
4.82\end{array}$ & $\begin{array}{l}\text { b** } \\
5.38\end{array}$ & $\begin{array}{l}a^{* *} \\
4.80\end{array}$ & $\begin{array}{l}\mathrm{b} * * \\
5.32\end{array}$ \\
\hline$\mu \mathrm{IU} / \mathrm{dL}$ & \pm 0.09 & \pm 0.95 & $\begin{array}{c} \pm 0.36 \\
(-28 \%)\end{array}$ & $\begin{array}{l} \pm 0.20 \\
(-6 \%)\end{array}$ & $\begin{array}{c} \pm 0.55 \\
(-16 \%)\end{array}$ & $\begin{array}{l} \pm 0.23 \\
(-6 \%)\end{array}$ & $\begin{array}{c} \pm 0.20 \\
(-16 \%)\end{array}$ & $\begin{array}{l} \pm 0.36 \\
(-7 \%)\end{array}$ \\
\hline Trigly- & 71.50 & 72.33 & 150.6 & $\begin{array}{l}b 8 * \\
88.67\end{array}$ & $9 \stackrel{a *}{4}$ & 83.16 & 170.5 & $\begin{array}{c}b^{*} \\
102.3\end{array}$ \\
\hline $\begin{array}{l}\text { cerides } \\
\mathrm{mg} / \mathrm{dL}\end{array}$ & \pm 4.76 & \pm 4.36 & $\begin{array}{c} \pm 4.86 \\
(111 \%)\end{array}$ & $\begin{array}{l} \pm 9.11 \\
(24 \%)\end{array}$ & $\begin{array}{l} \pm 4.17 \\
(31 \%)\end{array}$ & $\begin{array}{l} \pm 7.44 \\
(16 \%)\end{array}$ & $\begin{array}{c} \pm 8.76 \\
(138 \%)\end{array}$ & $\begin{array}{c} \pm 4.1 \\
(43 \%)\end{array}$ \\
\hline $\begin{array}{c}\text { Choles- } \\
\text { terol } \\
\mathrm{mg} / \mathrm{dL}\end{array}$ & $\begin{array}{c}135.7 \\
\pm 14.39\end{array}$ & $\begin{array}{c}136.6 \\
\pm 12.89\end{array}$ & $\begin{array}{c}\mathrm{a}^{* *} \\
382.8 \\
\pm 16.03 \\
(182 \%)\end{array}$ & $\begin{array}{c}b * * \\
182.0 \\
\pm 26.87 \\
(34 \%)\end{array}$ & $\begin{array}{c}32 \mathrm{a}^{* *} \\
\pm 14.12 \\
(140 \%)\end{array}$ & $\begin{array}{c}b^{*} \\
165.7 \\
\pm 11.60 \\
(22 \%)\end{array}$ & $\begin{array}{c}\mathrm{a}^{* *} \\
391.6 \\
\pm 16.32 \\
(188 \%)\end{array}$ & $\begin{array}{c}b * * \\
190.8 \\
\pm 25.38 \\
(41 \%)\end{array}$ \\
\hline HDL-c & 85.71 & 87.00 & $\begin{array}{c}\mathrm{a}^{* *} \\
50.67\end{array}$ & $\begin{array}{c}b^{* *} \\
64.00\end{array}$ & $\begin{array}{c}\mathrm{a}^{* *} \\
64.10\end{array}$ & $\begin{array}{c}b^{* *} \\
75.63\end{array}$ & $\begin{array}{c}a^{* *} \\
44.17\end{array}$ & $\begin{array}{c}\mathrm{b} * * \\
65.17\end{array}$ \\
\hline $\mathrm{mg} / \mathrm{dL}$ & \pm 8.75 & \pm 9.44 & $\begin{array}{c} \pm 6.71 \\
(-41 \%)\end{array}$ & $\begin{array}{c} \pm 2.76 \\
(-25 \%)\end{array}$ & $\begin{array}{c} \pm 7.68 \\
(-25 \%)\end{array}$ & $\begin{array}{c} \pm 3.91 \\
(-12 \%)\end{array}$ & $\begin{array}{c} \pm 6.11 \\
(-48 \%)\end{array}$ & $\begin{array}{c} \pm 2.78 \\
(-24 \%)\end{array}$ \\
\hline LDL-c & 35.66 & 35.16 & $\begin{array}{c}\mathrm{a}^{* *} \\
301.8\end{array}$ & 93.16 & $\begin{array}{c}\mathrm{a}^{* *} \\
242.5\end{array}$ & $\begin{array}{c}b^{*} \\
73.33\end{array}$ & 313.5 & $\begin{array}{c}\mathrm{b} * * \\
112.3\end{array}$ \\
\hline $\mathrm{mg} / \mathrm{dL}$ & \pm 6.44 & \pm 6.96 & $\begin{array}{c} \pm 9.49 \\
(757 \%) \\
\mathrm{a}^{* *}\end{array}$ & $\begin{array}{c} \pm 13.63 \\
(166 \%) \\
b * *\end{array}$ & $\begin{array}{c} \pm 8.19 \\
(592 \%) \\
a^{* *}\end{array}$ & $\begin{array}{c} \pm 7.00 \\
(109 \%) \\
b^{* *}\end{array}$ & $\begin{array}{c} \pm 10.84 \\
(796 \%) \\
a^{* *}\end{array}$ & $\begin{array}{c} \pm 13.41 \\
(220 \%) \\
b^{* *}\end{array}$ \\
\hline AI & -0.439 & -0.440 & 0.113 & -0.218 & -0.193 & -0.319 & 0.229 & -0.165 \\
\hline
\end{tabular}

Data are expressed as Mean \pm Standard Deviation $(n=10)$. Numbers between brackets show percentage change from the respective control value. a:significance vs control. b:significance vs respective DM, IRR and DM+IRR groups not given AM. *:significant at $\mathrm{P} \leq 0.05 ; * *$ :highly significant at $\mathrm{P} \leq 0.01$.

Atherogenic index $(\mathrm{AI})<0.11$ : low risk of cardiovascular disease (CVD); AI (0.11 to 0.21): medium risk of CVD; $\mathrm{AI}>$ 0.21 high risk of CVD. 
TABLE 4. Influence of Annona muricata(AM) on liver injury markers (ALT and AST activities) and kidney injury markers (urea and creatinine levels) in the serum of diabetic (DM), $\gamma$-irradiated (IRR) and diabetic- $\gamma$-irradiated (DM+IRR) rats.

\begin{tabular}{|c|c|c|c|c|c|c|c|c|}
\hline $\begin{array}{c}\text { Rat } \\
\text { Groups }\end{array}$ & Control & $\mathbf{A M}$ & DM & $\begin{array}{c}\text { DM } \\
+\mathbf{A M}\end{array}$ & IRR & $\begin{array}{c}\text { AM } \\
+ \text { IRR }\end{array}$ & $\begin{array}{c}\text { DM } \\
+ \text { IRR }\end{array}$ & $\begin{array}{c}\text { DM } \\
+ \text { +AM } \\
+ \text { IRR }\end{array}$ \\
\hline ALT & 21.92 & 21.52 & 51.42 & 22.36 & 25.85 & 23.36 & 30.50 & 22.66 \\
\hline $\mathrm{U} / \mathrm{L}$ & \pm 1.16 & \pm 1.22 & $\begin{array}{c} \pm 1.50 \\
(134 \%) \\
a^{* *}\end{array}$ & $\begin{array}{c} \pm 0.70 \\
(2 \%) \\
b^{* *}\end{array}$ & $\begin{array}{c} \pm 2.12 \\
(18 \%) \\
a^{* *}\end{array}$ & $\begin{array}{c} \pm 1.75 \\
(6.6 \%) \\
b^{*}\end{array}$ & $\begin{array}{c} \pm 1.41 \\
(39 \%) \\
a^{* *}\end{array}$ & $\begin{array}{c} \pm 1.96 \\
(3.4 \%) \\
b^{* *}\end{array}$ \\
\hline $\begin{array}{l}\text { AST } \\
\text { U/L }\end{array}$ & $\begin{array}{l}30.83 \\
\pm 3.18\end{array}$ & $\begin{array}{l}31.29 \\
\pm 4.37\end{array}$ & $\begin{array}{c}148.00 \\
\pm 15.31 \\
(380 \%) \\
a^{* *}\end{array}$ & $\begin{array}{c}69.25 \\
\pm 7.47 \\
(124 \%) \\
b^{* *}\end{array}$ & $\begin{array}{c}76.25 \\
\pm 5.60 \\
(147 \%) \\
a^{* *}\end{array}$ & $\begin{array}{c}50.50 \\
\pm 3.52 \\
(64 \%) \\
b^{* *}\end{array}$ & $\begin{array}{c}73.09 \\
\pm 1.81 \\
(137 \%) \\
a^{* *}\end{array}$ & $\begin{array}{c}49.80 \\
\pm 6.88 \\
(61 \%) \\
b^{* *}\end{array}$ \\
\hline $\begin{array}{l}\text { Urea } \\
\mathrm{mg} / \mathrm{dL}\end{array}$ & $\begin{array}{l}45.46 \\
\pm 2.58\end{array}$ & $\begin{array}{l}45.17 \\
\pm 2.34\end{array}$ & $\begin{array}{c}115.50 \\
\pm 1.83 \\
(154 \%) \\
\mathrm{a}^{* *}\end{array}$ & $\begin{array}{c}52.21 \\
\pm 6.75 \\
(15 \%) \\
b^{* *}\end{array}$ & $\begin{array}{c}63.83 \\
\pm 5.31 \\
(40 \%) \\
a^{* *}\end{array}$ & $\begin{array}{c}44.64 \\
\pm 2.08 \\
(-2 \%) \\
b^{* *}\end{array}$ & $\begin{array}{c}100.20 \\
\pm 3.76 \\
(120 \%) \\
a^{* *}\end{array}$ & $\begin{array}{c}71.83 \\
\pm 7.93 \\
(58 \%) \\
b^{* *}\end{array}$ \\
\hline $\begin{array}{c}\text { Creatinine } \\
\mathrm{mg} / \mathrm{dL}\end{array}$ & $\begin{array}{c}0.94 \\
\pm 0.07\end{array}$ & $\begin{array}{c}0.91 \\
\pm 0.09\end{array}$ & $\begin{array}{c}1.58 \\
\pm 0.14 \\
(68 \%) \\
a^{* *}\end{array}$ & $\begin{array}{c}1.03 \\
\pm 0.14 \\
(9 \%) \\
b^{*}\end{array}$ & $\begin{array}{c}1.09 \\
\pm 0.06 \\
(16 \%) \\
a^{*}\end{array}$ & $\begin{array}{c}1.01 \\
\pm 0.16 \\
(7 \%) \\
b^{*}\end{array}$ & $\begin{array}{c}1.65 \\
\pm 0.15 \\
(75 \%) \\
a^{* *}\end{array}$ & $\begin{array}{c}1.20 \\
\pm 0.16 \\
(27 \%) \\
b^{* *}\end{array}$ \\
\hline
\end{tabular}

Data are expressed as Mean \pm Standard Deviation $(n=10)$. Numbers between brackets showpercentage change from the respective control value. a:significance vs control. b:significance vs respective DM, IRR and DM+IRR groups not given AM. *: Significant at $\mathrm{P} \leq 0.05 ; * *$ : Highly significant at $\mathrm{P} \leq 0.01$

\section{Discussion}

Annona muricata L. is a popular fruit tree that has long been used in traditional medicine (Bidlaet al., 2004). In vivo and in vitro studies revealed that the leaves of Annona muricata possess anti-inflammatory (Ishola et al., 2014), hepatoprotective (Arthur et al., 2012), antioxidant (George et al., 2015; Coria-Tellez et al., 2016) and antidiabetic activities (Florence et al., 2014; Rahmi et al., 2016).

In the current study, the administration of the aqueous extract of $A$. muricata leaves to normal rats at a dose of $100 \mathrm{mg} / \mathrm{Kg}$ body weight for two weeks had no significant effect on the oxidative stress parameters of the liver, kidney and pancreas tissues. No significant changes were observed in serum glucose, insulin, lipid profile as well as liver and kidney functions. The results are in harmony with previous findings indicating that the oral administration of the aqueous extract of A. muricata leaves $(100 \mathrm{mg} / \mathrm{kg}$ bwt) during four weeks had no significant effect on SOD, catalase and MDA (Florence et al., 2014), glucose, lipid profile, ALT and AST activities and urea and creatinine levels (Arthur et al., 2011; Florence et al., 2014). The results support the suggestion that $A$. muricata leaves are practically non-toxic (Utomo et al.,2015).
In diabetic, $\gamma$-irradiated and diabetic- $\gamma$ irradiated rats, $A$. muricata treatment has significantly alleviated oxidative stress verified by a higher level of the antioxidant SOD activity and GSH content associated witha lower level of the lipid peroxidation end-product MDA inthe liver, kidney and pancreas compared to their relative levels in rats not treated with $A$. muricata. Experimental studies revealed that the decrease of antioxidants is caused by their increased utilization to neutralize free radicals together with a decreased synthesis (Yoshida et al., 2008; Matsunami et al., 2010) while lipid peroxidation arises by the interaction of $\mathrm{OH}$ radicals with unsaturated fatty acids (Bartsch and Nair, 2002; Spitz et al., 2004). The protective capacity of $A$. muricata is attributed to its role against ${ }^{\circ} \mathrm{OH}$ radicals (Baskar et al., 2007) and $\mathrm{H}_{2} \mathrm{O}_{2}$ (Muthu and Durairaj, 2015; George et al., 2015). The antioxidant potential of A. muricata might be ascribed to the presence of phytochemicals (Muthu and Durairaj, 2015) including luteolin, quercetin, epicatechingallate and emodin(George et al., 2015),nonenzymatic antioxidants such asVitaminE (Muthu and Durairaj, 2015), VitaminC and carotenoids (Usunomena and Paulinus, 2015), andenzymatic antioxidants including catalase, glutathione reductase and SOD (Muthu and Durairaj, 2015). The presence of ascorbic acid suppresses 
peroxidation in both aqueous and lipid region of cells (Dadheech et al., 2006). It traps peroxyl radicals before they can initiate lipid peroxidation and helps in the regeneration of Vitamin $\mathrm{E}$ (Chatterjee and Nandhini, 1991).The results are in harmony with previous findings indicating that the aqueous extractof $A$. muricataleaves protects against oxidative stress (Adewole and Ojewole, 2009; Olakunle et al., 2014) and enhancesSOD andcatalase activities, increases GSH content and reduces MDA (Moghadamtousi et al., 2015).

In diabetic, $\gamma$-irradiated and diabetic- $\gamma$-irradiated rats $A$. muricata treatment has significantly alleviated hyperglycemia, hypoinsulinemia and dyslipidemia. Hyperglycemia is generally the consequence of insulin deficiency (Akbarzadeh et al., 2007) due to oxidative stress and degeneration of pancreatic $\beta$-cells (Szkudelski, 2012), in addition to oxidative damage ofDNA(Nieman and Schalinske, 2011) causing depression of insulin synthesis(Kaneto et al.,1999). Dyslipidemia might be attributed to oxidative stress in liver tissues and alteration ofcholesterol, triglycerides, and lipoproteins synthesis (Zakim and Thomas, 2002). Moreover, oxidative stress induces damage to the receptors on the surface of many cells in the body which prevents the ingestion of LDL-C by endocytosis and might contribute to the increase of total cholesterol (Gent and Braakman, 2004). A muricata treatment has significantly reduced the levels of glucose,triglycerides and LDL-Csupporting the anti-hyperglycemic and anti-hyperlipidemic properties of A.muricata (Adeyemiet al., 2008a).

The results corroborate that the oral administration of the aqueous extract of $A$. muricata leaves reduced glucose level (Florence et al., 2014). The modulatory role of A. muricata on hyperglycemia and hypoinsulinemia might be attributed to its role in the protection of pancreatic $\beta$-cells against oxidative stress (Florence et al.,2014) and regeneration of $\beta$-cells (Adeyemi et al., 2008b). The antioxidant effect is probably mediated by the inactivation of NF$\kappa \mathrm{B}$ and the consequent decrease in formation of nitric oxide (NO),a mediator of islet beta cell damage (Wolff et al., 1991). Supporting this postulation, histological examination of pancreas tissues revealed that A.muricata protected and preserved pancreatic $\beta$-cell integrity in parallel to a significant decrease of blood NO (Adewole and Caxton-Martins, 2006).Furthermore, the leaves of A.muricata contain magnesium (Mg),
Chromium (Cr) and zinc (Zn) (Usunomena and Paulinus, 2015). Magnesium helps insulin secretion from the beta cells (Gommers et al., 2016) and thusregulates insulin level.Chromiumis an essential mineral that is thought to be necessary for normal glucose and lipid homeostatsis(Cefalo and $\mathrm{Hu}, 2004)$. In this line, Zhang et al. (2014) suggested that chromium improves blood glucose in diabetic rats by activating insulin synthesis in islet.On the other hand (Emdin et al., 1980) found that zinc plays an important role in insulin production in the $\beta$-cell.

Also the hypoglycemic activity of A.muricata leaves might be attributed to the presence of certain flavonoids that have the ability to inhibit $\alpha$-glucosidase (Rahmiet al., 2016) thus decreasing carbohydrate metabolism and glucose absorption (Hardokoet al., 2015). Additionally, the glycemic index and the glycemic load of A.muricata were found to be low in A. muricata, which supports its hypoglycemic potential (Passos et al., 2015).

In the current study, the improvement of dyslipidemia in A. muricata-treated rats are in agreement with the findings of Ahalya et al. (2014) suggesting that A. muricata reduces cholesterol and triglyceride levels. The results are also in harmony with the findings of Adewole and Ojewole (2009) and Florence et al. (2014) who reported that the oral administration of the leaf aqueous extractof $A$. muricata $(100 \mathrm{mg} / \mathrm{kg} /$ day) to diabetic rats during four consecutive weeks has significantly decreased the elevated total cholesterol, triglycerides and LDL-C. Moreover, Annona muricata leaves contain saponins, known to produce inhibitory effect on inflammation (Just et al., 1998). Saponins as a class of natural products are involved in complexation with cholesterol to form pores in cell membrane bilayers (Francis et al., 2002), and as such may be used as anticholesterol agents or cholesterol lowering agents.

In diabetic, $\gamma$-irradiated and diabetic- $\gamma$ irradiated rats, theelevationof serum AST and ALT activities indicate liver injury (Botros and Sikaris, 2013) while the increase of urea and creatinine indicates kidney damage (Siew et al., 2011). In the current study, liver and kidney injury appears to be the consequence of oxidative stress verified by the increase of MDA associated with a decrease of SOD activity and GSH content. Increased lipid peroxidation of cell membrane causes alteration of cell membrane permeability. A. muricata treatment has significantly alleviated liver and kidney dysfunction. The results corroborate the Egypt. J. Rad. Sci. Applic., Vol. 30, No.1(2017) 
hepatoprotective role of $A$. muricata(Adewole and Ojewole, 2009) and that A.muricata aqueous leaf extract could restore liver function toward normal levels (Arthur et al., 2012).

Experimental evidence suggests that proinflammatory cytokines such asInterleukin- $1 \beta$ (IL-1 $\beta$ ) and Tumor necrosis factor-alpha (TNF- $\alpha$ ) play an important role in liver (Christiansen et al., 2007) and kidney damage (Chan et al., 2010; Hamid et al., 2012). Thus, in the current study, the amelioration of liver and kidney functions in A. muricata- treated rats might be attributed to its anti-inflammatory properties (Ishola et al., 2014) and attenuation of TNF- $\alpha$ and IL-1 $\beta$ protein expression (Chan et al., 2010; Hamid et al., 2012). Moreover, the amelioration of liver and kidney functions appears to be the consequence of the improvement of oxidative stress.

The present study demonstrates that $A$. muricata ameliorates oxidative stress in the liver, kidney and pancreas, associated with improvement of hyperglycemia, hypoinsulinemia, hyperlipidemia besides improvement of both liver and kidney functions which could be attributed to the synergistic relationship between the different elements found in the leaves of A.muricata. It could be concluded that supplementation of A.muricata could be beneficial in ameliorating liver and kidney functions and correcting metabolic variations associated with oxidative stress in the liver, kidney and pancreas.

\section{References}

Adewole, S.O and Ojewole, J.A. (2008) Protective effects of Annona muricata Linn. (Annonaceae) leaf aqueous extract on serum lipid profiles and oxidative stress in hepatocytes of streptozotocin-treated diabetic rats. African journal of traditional, complementary and alternative medicines 6(1): 30-41.

Adewole, S.O and Caxton-Martins EA (2006) Morphological Changes and Hypoglycemic Effects of Annona Muricata Linn. (Annonaceae) Leaf Aqueous Extract on Pancreatic B-Cells of Streptozotocin-Treated Diabetic Rats. African Journal of Biomedical Research,9:173 - 187

Adeyemi, D.O., Komolafe, O.A., Adewole, S.O., Obuotor, E.M. and Adenowo, T.K. (2008 a)Anti hyperlipidemic activities of Annona muricata (Linn). Afr J Trad Complement Altern Med 6(1): 62-69.
Adeyemi, D.O., Komolafe, O.A., Adewole, S.O., Obuotor, E.M. and Adenowo T.K. (2008 b) Effects of Annona muricata(Linn) on the morphology of pancreatic islet cells of experimentally-induced diabetic Wistar rats. Int.J. Altern Med.5(2): 1-8

Ahalya, B., Shankar, K.R. and Kiranmayi, G. (2014) Exploration of anti-hyperglycemic and hypolipidemic activities of ethanolic extract of Annona muricatabark in alloxan-induced diabetic rats. Int J Pharm Sci Rev Res. 25: 21-27

Akbarzadeh, A., Norouzian, D., Mehrabi, M.R., Jamshidi, S., Farhangi, A., Allah A., Mofidian, S. and Lame Rad B. (2007) Induction of diabetes by streptozotocin in rats. Indian JClinBiochem. 22(2):60-64.

American Diabetes Association (2009) Diagnosis and Classification of Diabetes Mellitus. Diabetes Care 32(Suppl 1): S62-S67.

Arthur, F.K., Terlabi, E.O., Larbie, C. and Woode, E. (2012) Evaluation of hepatoprotective effect of aqueous extract of Annona muricata(Linn.) leaf against carbon tetrachloride and acetaminopheninduced liver damage.J Nat Pharm.3:25-30.

Arthur, F.K., Woode, E., Terlabi, E.O. and Larbie, C. (2011) Evaluation of acute and subchronic toxicity of Annona Muricata (Linn.) aqueous extract in animals. Eur J Exp Biol. 1 (4):115-124

Asare, G.A., Afriyie, D., Ngala, R.A., Abutiate, H., Doku, D., Mahmood, S.A. and Rahman, H. (2015) Antiproliferative activity of aqueous leaf extract of AnnonamuricataL. on the prostate, BPH-1 cells, and some target genes. Integr Cancer Ther.14:65-74.

Bartsch, H. and Nair, J. (2002) Potential role of lipid peroxidation derived DNA damage in human colon carcinogenesis: studies on exocyclic base adduct as stable oxidative stress markers. Cancer Detect Prev. 26(4):308-312.

Baskar, R., Rajeswari, V. and Kumar, T.S. (2007) In vitro antioxidant studies in leaves of Annona species. Indian J. Exp. Biol. 45: 480-485.

Beutler, E., Duron, O. and Kelly, B.M. (1963)Improved method for the determination of blood glutathione. J. Lab. Clin. Med. 61:882-888.

Bidla, G., Titanji, V., Joko, B., El-Ghazali, G., Bolad, A. and Berzins, K. (2004) Antiplasmodial activity of seven plants used in African folk medicine. Indian J Pharmacol 6: 245-246. 
Botros, M. and Sikaris, A.K. (2013) The De Ritis Ratio: The Test of Time. Clin Biochem review 34(3): 117-130

Cefalu, W.T. and Hu., F.B. (2004) Role of chromium in human health and in diabetes. Diabetes Care 27 (11): 2741-2751.

Chan, P., Ah, R. and Mh, K. (2010) Anti-arthritic activities of Annona muricata L Leaves extract on complete Freund's adjuvant (CFA)-induced arthritis in rats. Planta Med.76: P166.

Chatterjee, I. B. and Nandhini, A. (1991) Ascorbic acid; a scavenger of oxyradicals. Ind $J$ Biochem Biophy. 28: 233-236.

Christiansen, H., Sheikh, N., Saile, B., Reuter, F., RaveFränk, M., Hermann, R.M., Dudas, J., Hille, A., Hess, C.F.and Ramadori, G.(2007) X-Irradiation in rat liver: consequent upregulation of hepcidin and downregulation of hemojuvelin and ferroportin-1 gene expression. Radiology 242:189-197

Clark, P.M.S.and Hales, C.N. (1994): How to measure plasma insulin. Diabetes/Metabolism Reviews, 10:79-90.

Coria-Tellez, A.V., Montalvo - Gonzalez, E., Yahia, E.M. and Obledo-Vazquez, E.N. (2016)Annona muricata: A comprehensive review on its traditional medicinal uses, phytochemicals, pharmacological activities, mechanisms of action and toxicity. Arabian Journal of Chemistry (2016). doi:http://dx.doi.org/10.1016/j.arabjc.2016.01.004

Dadheech, G., Mishra, S., Gautam, S. and Sharma, P. (2006) Oxidative stress, $\alpha$-tocopherol, ascorbic acid and reduced glutathione status in Schizophrenics. Indian J. Clin. Biochem.21: 34-38.

Dobiasova, M. (2006) AIP-atherogenic index of plasma as a significant predictor of cardiovascular risk: from research to practice. VnitrLek 52(1): 64-7.

Emdin, S.O., Dodson, G.G., Cutfield, J.M. and Cutfield, S.M. (1980) Role of Zinc in Insulin Biosynthesis Some Possible Zinc-Insulin Interactions in the Pancreatic B-cell. Diabetologia 19, 174-182.

Erejuwa, O.O., Sulaiman, S.A., Wahab, M.S.A., Sirajudeen, K.N.S.Salleh, M.S.M. and Gurtu, S. (2011) Glibenclamide or metformin combined with honey improves glycemic control in streptozotocininduced diabetic rats. Int. J. Biol. Sci.7(2): 244-252.

Florence, N.T., Benoit, M.Z., Jonas, K., Alexandra, T., Désiré, D.D.P., Pierre, K. and Théophile, D. (2014) Antidiabetic and antioxidant effects of Annona muricata (Annonaceae), aqueous extract on Streptozotocin-induced diabetic rats.J Ethnopharmacol.151:784-790.
Fossati, P.and Prencipe, L. (1982) Serum Triglycerides Determination Colorimetrically with an Enzyme Produce Hydrogen Peroxide.Clin Chem, 28, 10: 2077-2080.

Francis, C., George, G., Zohar, K., Harinder, P.S., Makhar, L.M. and Klaus, B.(2002) The biological action of saponins in animal system: a review. British J. Nutrition 88(6): 587-605

Friedewald, W.T., Levy, R.T. and Frederickson, D.S. (1972) Estimation of the concentration of lowdensity lipoprotein cholesterol in plasma without use of the preparative ultracentrifuge.Clin Chem. 18: 499-502.

Gandhi, G.R. and Sasikumar, P. (2012) Antidiabetic effect of Merremiaemarginata Burm. F. in streptozotocin induced diabetic rats. Asian Pacific J. Tropl Biomed.2(4): 281-286.

Gent, J. and Braakman, I. (2004) Low-density lipoprotein receptor structure and folding. Cell $\mathrm{Mol}$ Life Sci. 61 (19-20): 2461-70

George, V.C., Kumar, D.N., Suresh, P.and Kumar R.A. (2015) Antioxidant, DNA protective efficacy and hplc analysis of Annonamuricata(soursop) extracts.J Food SciTechnol 52: 2328-2335.

Gommers, L.M.M., Hoenderop, J.G.J., Bindels, R.J.M. and de Baai, J.H.F (2016) Hypomagnesemia in Type 2 Diabetes: A Vicious Circle? Diabetes 65(1): 3-13

Hamid, R.A., Foong, C.P., Ahmad, Z. and Hussain, M.K. (2012) Antinociceptive and anti-ulcerogenic activities of the ethanolic extract of Annonamuricata leaf. Rev Bras Farmacogn. 22: 630-641

Hardoko, H., Halim, Y. and Wijoyo, S. (2015) In vitro antidiabetic activity of "Green Tea" soursop leaves brew through $\alpha$-glucosidase inhibition. Int J Pharm Tech Res. 8 (1), 30-37.

Henry, RJ., Cannon, D.C. and Winkelman, W. (1974) Clinical Chemistry Principles and Techniques, $11^{\text {th }}$ ed Harper and Row, pp 1629

Ishola, I.O., Awodele, O., Olusayero, A.M.a nd Ochieng, C.O. (2014) Mechanisms of analgesic and antiinflammatory properties of AnnonamuricataLinn. (Annonaceae) fruit extract in rodents. $J$ Med Food 17:1375-1382

Just, M.J., Recio, M.C., Giner, R.M., Cuellar, M.J., Manez, S., Bilia, A.R. and Rios, J.L. (1998) Antiinflammatory activity of unusual lupinesaponins from Bupleurumfruticescens. Plant Med. 64: 04-407.

Egypt. J. Rad. Sci. Applic., Vol. 30, No.1(2017) 
Kakkar, P., Das, B.and Viswanathan, P.N. (1984) A modified spectrophotometric assay of superoxide dismutase. Ind J BiochemBiophys 21:130-132

Kaneto, H., Kajimoto, Y., Miyagawa, J., Matsuoka, T., Fujitani, Y.; Umayahara, Y.; Hanafusa, T., Matsuzawa, Y.; Yamasaki, Y.and Hori, M. (1999) Beneficial Effects of Antioxidants in Diabetes. Possible Protection of Pancreatic $\beta$-Cells Against Glucose Toxicity. Diabetes 48: 2398-2406

Matsunami, T., Sato, Y. and Yukawa, M. (2010) Oxidative stress and gene expression of antioxidant enzymes in the streptozotocin-induced diabetic rats under hyperbaric oxygen exposure. Int.J. Clin.Exp. Pathol. 3(2): 177-188

Moghadamtousi, S.Z., Rouhollahi, E., Hajrezaie, M., Karimian, H., Abdulla, M.A. and Kadir, H.A. (2015) Annona muricataleaves accelerate wound healing in rats via involvement of hsp70 and antioxidant defence. Int $J$ Surg18:110-117.

Muthu, S. and Durairaj, B. (2015) Evaluation of antioxidant and free radical scavenging activity of Annona muricata. Eur J ExpBiol 5(3): 39-45

Nieman, K.M. and Schalinske, K.L. (2011) Insulin administration abrogates perturbation of methyl group and homocysteine metabolism in streptozotocin-treated type 1 diabetic rats. $\mathrm{Am} \mathrm{J}$ PhysiolEndocrinolMetab. 301:E560-E565.

Olakunle, S., Onyechi, O. and James, O. (2014) Toxicity, anti-lipid peroxidation, in vitro and in vivo evaluation of antioxidant activity of Annonamuricataethanol stem bark extract. Am J Life Sci.2: 271-277.

Passos, T.U., Alves, H., Sampaio, D.C., Olgane, M., Sabry, D., Luisa, M.and Lima, D.O. (2015) Glycemic index and glycemic load of tropical fruits and the potential risk for chronic diseases. Food SciTechnol Int. 35 (1), 66-73.

Patel, S. and Patel, J.K. (2016) A review on a miracle fruits of Annonamuricata. $J$. PharmacognPhytochem. 5(1): 137-148

Patton, C.J.and Crouch, S.R. (1977) Spectrophotometric and Kinetics Investigation of the Berthelot Reaction for the Determination of Ammonia. Anal Chem. 49(3): 464-469.

Rahmi, E., Wahyuni, W.T., Darusman, L.K. and Suparto, I.H. (2016) Combination of ethanolic extract of $\alpha$-glucosidase inhibitory activity of Phaleriamacrocarpa (Scheff.) boerl fruits and Annonamuricata Linn leaves. Trad Med. 21(2):63-68
Reitman, S. and Frankel, S. (1957) Colorimetric method of the determination of serum transaminase. $\mathrm{Am} \mathrm{J}$ Clin Path. 28: 56-60.

Richmond, N. (1973) Colorimetric determination of total cholesterol and high density lipoprotein cholesterol (HDL-C). Clin Chem. 19(12): 1350- 1356

Saada, H.N., Eltahawy, N.A., Morcos, N.Y.S. and Hammad, A.S. (2016) Gamma amino butyric acid attenuates liver and kidney damage associated with insulin alteration in $\gamma$-irradiated and streptozotocintreated rats. Arab J. of Nuclear Science and Applications 94 (1): 138-150

Siew, E.D., Ware, L.B. and Ikizler, T.A. (2011): Biological Markers of Acute Kidney Injury. J. Am. Soc.Nephrol. 22: $810-820$

Spitz, D.R., Azzam, E.I., Li, J.J. and Gius, D. (2004) Metabolic oxidation/reduction reactions and cellular responses to ionizing radiation: A unifying concept in stress response biology. Cancer and Metastasis Reviews 23: 311-322

Sun, J., Chen, Y., Li, M. and Ge, Z. (1998) Role of antioxidant enzymes on ionizing radiation resistance. Free Radic Biol Med. 24: 586-593.

Szkudelski, T. (2012) Streptozotocin-nicotinamideinduced diabetes in the rat. Characteristics of the experimental model.Exp. Biol. Med. 237: 481-490.

Trinder, P. (1969) Enzymatic colorimetric determination of glucose. Ann ClinBiochem. 6, 2: 24-27.

Usunomena, U. and Paulinus, O.N. (2015) Phytochemical analysis and mineral composition of Annonamuricata leaves. IntJ of research and current development (IJRCD), 1(1): 38-42.

Utomo, A.W., Susilaningsih, N. and Armalina, D. (2015) Acute toxicity test of soursop leaves (Annonamuricata) on liver and kidney of Switzerland mice.Sains Medika Journal of medicine and health, $6(2)$

Wang, J.Y., Zhu, C., Qian, T.W., Guo, H., Wang, D.D., Zhang, F. and Yin, X. (2015) Extracts of black bean peel and pomegranate peel ameliorate oxidative stress-induced hyperglycemia in mice. ExpTher Med. 9 (1): 43-48.

Wolff, S.P., Jiang, Z.Y. and Hunt, J.V. (1991) Protein glycation and oxidative stress in diabetes mellitus and ageing. Free RadicBiol Med. 10: 339-352.

Yoshida, S., Hashimoto, T., Kihara, M., Imai, N., Yasuzaki, H., Nomura, K., Kiuchi, Y., Ishigami, T.,

Egypt. J. Rad. Sci. Applic., Vol. 30, No.1(2017) 
Hirawa, N.,Toya, Y., Kitamura, H. and Umemura, S. (2008) Urinary oxidative stress markers closely reflect the efficacy of Candesartan treatment for diabetic nephropathy. Nephron ExpNephrol. 111:20-30.

Yoshioka, T., Kawada, K., Shimada, T.and Mori, M. (1979) Lipid peroxidation in maternal and cord blood and protective mechanism against activated oxygen toxicity in the blood. Am. J.Obstet Gynecol. 135: $372-376$.
Zakim, D. and Thomas, D. (2002) Hepatology: A Textbook of Liver Disease (4 ${ }^{\text {th }}$ edition).

Zhang, Q., Xiao, X., Li, M., Li, W., Yu, M., Zhang, H., Ping, F., Wang, Z., Zheng, J. and Xiang, H. (2014):miR-375 and miR-30d in the Effect of Chromium-Containing Chinese Medicine Moderating Glucose Metabolism. Journal of Diabetes Research,Volume 2014: Article ID 862473. http://dx.doi.org/10.1155/2014/862473

(Received 30/5 /2017; acecpted 5 / 7/ 2017)

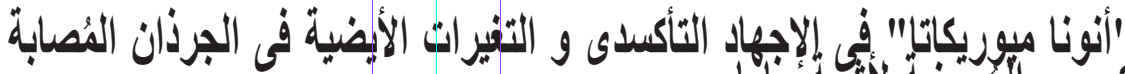

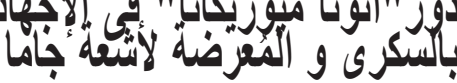

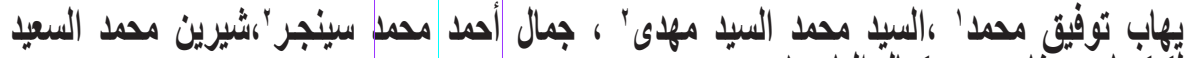

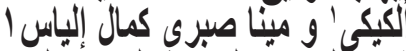

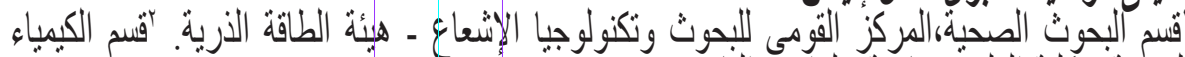

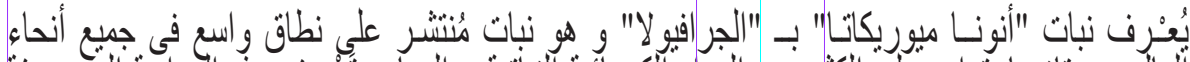

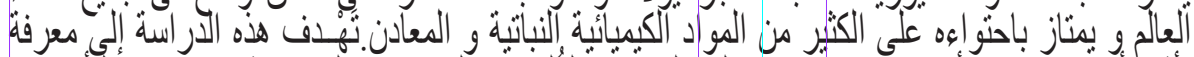

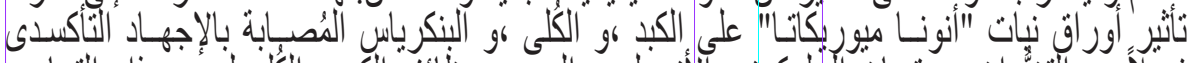

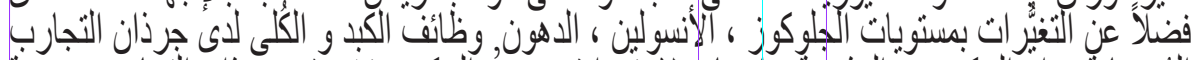

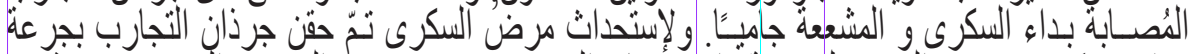

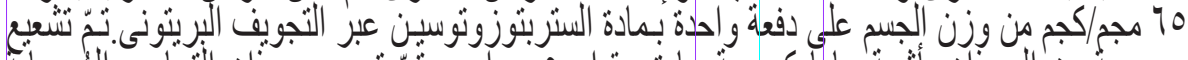

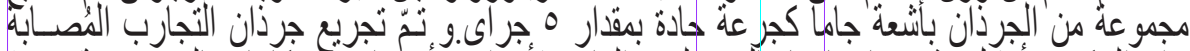

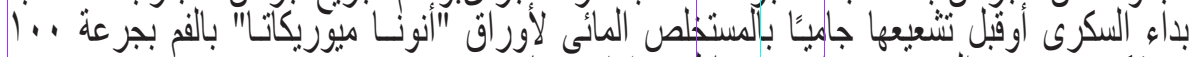

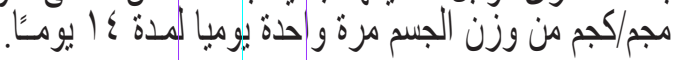

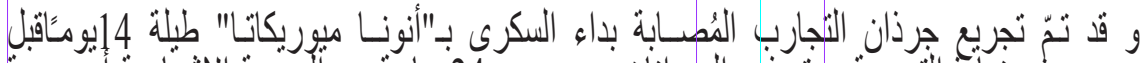

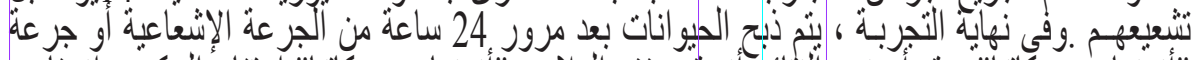

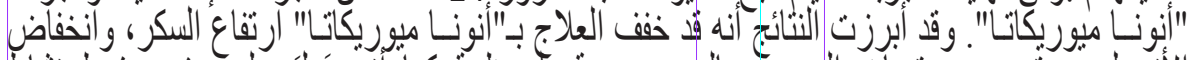

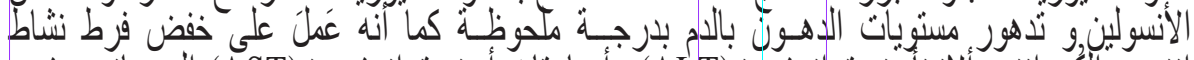

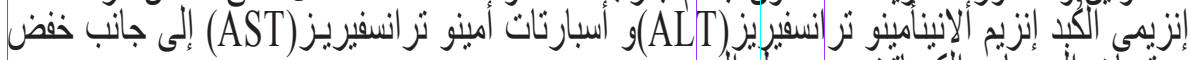

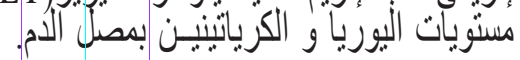

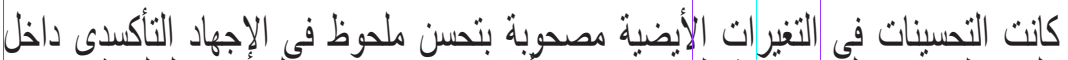

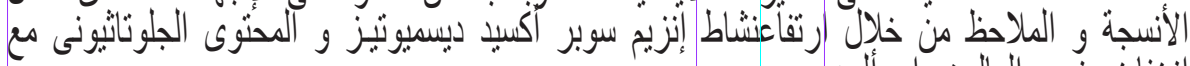
انخفاض نسب المالون داي ألاديا.

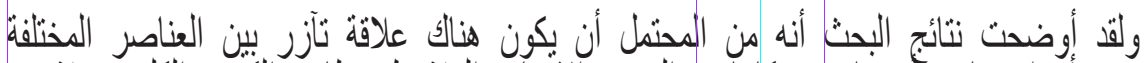

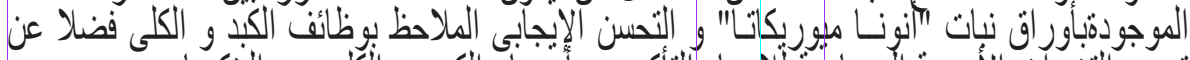

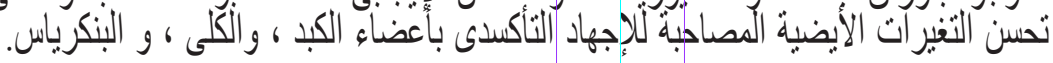

